Linear Analysis and Verification Suite for Edge Turbulence

J.R. Myra, M. Umansky

April 25, 2008 
This document was prepared as an account of work sponsored by an agency of the United States government. Neither the United States government nor Lawrence Livermore National Security, LLC, nor any of their employees makes any warranty, expressed or implied, or assumes any legal liability or responsibility for the accuracy, completeness, or usefulness of any information, apparatus, product, or process disclosed, or represents that its use would not infringe privately owned rights. Reference herein to any specific commercial product, process, or service by trade name, trademark, manufacturer, or otherwise does not necessarily constitute or imply its endorsement, recommendation, or favoring by the United States government or Lawrence Livermore National Security, LLC. The views and opinions of authors expressed herein do not necessarily state or reflect those of the United States government or Lawrence Livermore National Security, LLC, and shall not be used for advertising or product endorsement purposes.

This work performed under the auspices of the U.S. Department of Energy by Lawrence Livermore National Laboratory under Contract DE-AC52-07NA27344. 


\title{
Linear Analysis and Verification Suite for Edge Turbulence
}

\author{
J. R. Myra \\ Lodestar Research Corp., 2400 Central Ave. P-5, Boulder, Colorado 80301
}

M. Umansky,

Lawrence Livermore National Laboratory, Livermore, CA 94550

April 2008

unpublished 


\title{
Linear Analysis and Verification Suite for Edge Turbulence
}

\author{
J. R. Myra \\ Lodestar Research Corp., 2400 Central Ave. P-5, Boulder, Colorado 80301
}

M. Umansky, Lawrence Livermore National Laboratory, Livermore, CA 94550

\begin{abstract}
The edge and scrape-off-layer region of a tokamak plasma is subject to well known resistive and ideal instabilities that are driven by various curvature- and sheathrelated mechanisms. While the boundary plasma is typically strongly turbulent in experiments, it is useful to have computational tools that can analyze the linear eigenmode structure, predict quantitative trends in growth rates and elucidate and the underlying drive mechanisms. Furthermore, measurement of the linear growth rate of unstable modes emerging from a known, established equilibrium configuration provides one of the few quantitative ways of rigorously benchmarking large-scale plasma turbulence codes with each other and with a universal standard. In this report, a suite of codes that can describe linearized, nonlocal (e.g. separatrix-spanning) modes in axisymmetric (realistic divertor), toroidal geometry is discussed. Examples of several benchmark comparisons are given, and future development plans for a new eigenvalue edge code are presented.
\end{abstract}




\section{Introduction}

In this report, we describe a suite of codes that can be employed to study the linear structure and properties of eigenmodes in the boundary plasma of an axisymmetric tokamak. Here by boundary plasma, we mean the edge (closed flux surface) region as well as the scrape-off-layer (SOL) (open flux surface) region. The code suite consists of the BOUT code ${ }^{1,2}$ as well as a new edge eigenvalue code, called 2DX.

Although the boundary plasma is typically strongly turbulent in experiments, ${ }^{3}$ it is useful to have computational tools that can analyze the linear eigenmode structure, predict quantitative trends in growth rates and elucidate and the underlying drive mechanisms. Thus, linear codes are useful for modeling, theory, and experimental analysis. One possible application could be to supplement existing magnethydrodynamic (MHD) codes but with a two-fluid or kinetic model appropriate to the edge. Additionally, theoretical work in the boundary plasma has shown that there is, in fact, a correspondence principle ${ }^{4}$ relating the properties of linear modes to strongly nonlinear coherent structures such as blob filaments. ${ }^{5}$

Of course, to quantitatively predict turbulent transport in the boundary plasmas, comprehensive nonlinear codes are required. The tokamak edge physics community is becoming increasingly reliant on large-scale-simulation for this task. Rigorous verification and validation $(\mathrm{V} \& \mathrm{~V})$ of edge codes is thus critical. ${ }^{6}$ Measurement of the linear growth rate of unstable modes emerging from a known, established equilibrium configuration provides one of the few quantitative ways of rigorously benchmarking large-scale plasma turbulence codes with each other and with a universal standard. The code suite discussed here has been developed in an effort to create a trusted community standard for this purpose.

Tokamak edge and SOL physics is complicated by the magnetic geometry inherent in diverted configurations. The BOUT code, 1,2 is the only three-dimensional (3D) fluid turbulence code that we are aware of, that treats the full axisymmetric (realistic divertor), toroidal geometry. This is important because modes which span the separatrix or last-closed-surface (LCS) can be critical for the exhaust of particles and heat from the edge region into the SOL. Furthermore, momentum transport across the LCS by such modes is important in the theory of sheared flows and turbulence-driven plasma rotation. ${ }^{7}$ Finally, the transport of parallel current by edge-localized modes ${ }^{8}$ (ELMs) from the edge into the SOL is of contemporary interest. ${ }^{9}$ Treatment of these effects require "global” edge codes with realistic geometry capabilities. Quasilinear theory based on the linear mode structure provides a useful measure of these fluxes in many cases (at least their relative size, when saturation amplitudes are not known) and is another possible application. 
The plan of our report is as follows. In Sec. II we describe the codes in our test suite. Section III describes the physics models used for verification. Section IV is the main body of our report. Here we present a series of verification "benchmark" comparisons using our code suite. Section V presents some additional development work on the 2DX code, including a proof-of-principle test for including kinetic physics, and a discussion of future plans. A summary is given in Sec. VI. Some details of the coordinate systems, 2DX code architecture and implementation are in Appendices.

\section{The code suite}

\section{A. The BOUT code}

The BOUT code is a 3D fluid turbulence code which simulates plasma evolution in the boundary plasma.1,2 BOUT handles complex magnetic topologies and implement full toroidal divertor geometry, including $\mathrm{X}$-points in both single and double null configurations. In a single null configuration, BOUT evolves plasma in both the closed flux surface region, as well as in the open field line regions of the main and private (i.e. divertor region) SOLs. BOUT is a "global" code (includes full radial mode structure with radially varying geometry and plasma profiles). The fluid equations describing the plasma are evolved by an implicit time step advancement scheme. The BOUT code is at a mature stage in its development, and details have been described elsewhere.

In the present work, we exploit the capability of BOUT to turn off any term in its comprehensive physics model. Thus the code can be employed to carry out validation benchmark tests in simple (even analytically tractable) limits. For the linear growth rate studies considered here, BOUT is run with all nonlinear terms turned off. Growth rates and corresponding wavenumbers are measured from the amplitude of the fastest growing mode.

\section{B. The 2DX code}

The 2DX code is a newly developed linear eigenvalue code for the boundary plasma. Like BOUT it is a global code. Linearity and toroidal axisymmetry allow a given toroidal mode to be studied in isolation, i.e. modes are assumed to vary like

$$
\Phi \sim \exp \left(-\mathrm{i} \omega \mathrm{t}+\mathrm{in}_{\zeta} \zeta\right)
$$

where $\omega$ is the complex frequency, $\mathrm{n}_{\zeta}$ is the toroidal mode number and $\zeta$ is the geometrical toroidal angle. In Phase I of development, the 2DX code has implemented a simple fluid model, the resistive ballooning equations discussed in the next section. A more complete fluid model, equivalent to that in BOUT, is in the development plan. Additionally, kinetic extensions are discussed in Sec. IV. 
The magnetic geometry is also being implemented in 2DX following a phased plan. In the initial phase, we implemented the simplest geometry: a circular flux surface, small aspect ratio limit where the plasma and magnetic equilibrium is analytical. Even in this stage of development, rather rich physics is still possible. Both an edge and SOL region can be described with all their inherent instabilities, and numerical methods for coupling the edge and SOL regions can be tested. Work has recently been completed to implement full toroidal divertor geometry as in BOUT, and will be touched on briefly here.

The phased development of the 2DX code began with the edge region and a progression of tests through increasingly more complex equations and geometry. In the edge region, where periodic boundary conditions were implemented, it was possible to test the following modes: (i) resistive interchange, (ii) shearless resistive ballooning, and (iii) sheared resistive ballooning. 10 In field-line-following coordinates, periodic boundary conditions are not trivial, and must take account of the so-called ballooning transformation physics. ${ }^{11}$ This is discussed in Appendix A. In the SOL region, after implementing sheath boundary conditions, we tested (iv) the Nedospasov mode, 12,13 and (v) the conducting wall mode. ${ }^{14}$ In the last case, a slightly different set of model equations needed to be employed.

The basic algorithm of the 2DX code is as follows. We project the unknown eigenfunction onto a 2D grid (radial, poloidal) using basis modes (e.g. Fourier) or spatial discretization. An abstract vector $\xi$ is assumed to contain the field variables on the $2 \mathrm{D}$ grid or basis, e.g. for the two-field resistive ballooning model discussed in Sec. III, $\xi=$ $(\Phi, \mathrm{n})$ where $\Phi$ is the electrostatic potential and $\mathrm{n}$ is the plasma density. The equations and boundary conditions of the physics model are cast as operators in this space. A matrix operator $\mathrm{M}$ contains blocks for the interactions of the fields. Thus the eigenvalue problem is rendered in the form

$$
\mathrm{M} \xi=\gamma \xi
$$

where $\gamma=\operatorname{Im}(\omega)$. Expressing the blocks of M explicitly, we have

$$
\left(\begin{array}{ll}
M_{11} & M_{12} \\
M_{21} & M_{22}
\end{array}\right)\left(\begin{array}{l}
\Phi \\
n
\end{array}\right)=\gamma\left(\begin{array}{l}
\Phi \\
n
\end{array}\right)
$$

Standard eigen-system solvers are employed to obtain the eigenvalues and eigenvectors of $\mathrm{M}$. For a radial and poloidal resolution of $\left(\mathrm{n}_{\mathrm{x}}, \mathrm{n}_{\mathrm{y}}\right)$ respectively, and a problem with $\mathrm{p}$ fields (here $p=2$ ) the problem size is $n=p n_{x} n_{y}$. M is an $n \times n$ matrix which can get quite large for high resolution cases with many fields. $\mathrm{M}$ can be full or sparse depending on the basis set and method selected.

In addition to the flexibility in numerical methods, most of coding has been implemented in two coordinate systems: geometrical-angle (GA) and field-line-following (FLF) coordinates. Modular code architecture allows this flexibility with very little extra 
effort. Details are discussed in Appendix A. Have both coordinate systems provides another useful check of the results.

\section{Verification models}

\section{A. The resistive ballooning model}

Most of the initial tests for 2DX code development and for verification of 2DX and BOUT have been done in the 2-field $(\Phi, \mathrm{n})$ resistive ballooning model.

$$
\begin{aligned}
\frac{\mathrm{c}^{2}}{4 \pi \mathrm{v}_{\mathrm{a}}^{2}} \frac{\partial}{\partial \mathrm{t}} \nabla_{\perp}^{2} \Phi & =-\sigma_{\|} \nabla_{\|}^{2} \Phi+\frac{2 \mathrm{c}}{\mathrm{B}} \mathbf{b} \times \kappa \cdot \nabla\left(\mathrm{nT}_{0}\right) \\
\frac{\partial \mathrm{n}}{\partial \mathrm{t}} & =-\frac{\mathrm{c}}{\mathrm{B}} \mathbf{b} \times \nabla \Phi \cdot \mathbf{e}_{\mathrm{x}} \frac{\partial \mathrm{n}_{0}}{\partial \mathrm{x}}
\end{aligned}
$$

Here, $v_{a}$ is the Alfven velocity, $c$ is the speed of light, $\sigma_{\|}$is the Spitzer conductivity parallel to the magnetic field $\mathbf{B}=\mathrm{Bb}, \kappa$ is the curvature, and $\mathrm{n}_{0}$ and $\mathrm{T}_{0}$ are the equilibrium density and temperature. For a plasma slab at the outboard midplane of the torus, the curvature is taken as $\kappa=-\mathbf{e}_{\mathrm{X}} / \mathrm{R}$ where $\mathrm{R}$ is the major radius, and $\mathbf{e}_{\mathrm{X}}=\mathbf{e}_{\mathrm{R}}$ is the outward radial direction. For an annular shell of edge plasma the curvature is $\kappa=-\mathbf{e}_{\mathrm{X}}$ $\cos \theta / \mathrm{R}$ where $\theta$ is the poloidal angle, and $\theta=0$ at the outboard midplane.

The boundary conditions for the model are: periodic in $\theta$ in the closed flux surface region, and sheath boundary conditions in the SOL. The latter implies that at the sheath entrance (boundary of the domain)

$$
\mathrm{J}_{\|} \equiv-\sigma_{\|} \nabla_{\|} \Phi=-\mathrm{s}_{\mathrm{n}} \frac{\mathrm{nec}_{\mathrm{s}}}{\mathrm{T}_{0}}(\mathrm{e} \Phi-3 \mathrm{~T})
$$

where $\mathrm{s}_{\mathrm{n}}=\mathbf{n} \cdot \mathbf{b} /|\mathbf{n} \cdot \mathbf{b}|= \pm 1$, and $\mathbf{n}$ is the outward normal from the surface (i.e. pointing into the plasma). When $\mathrm{T}$ is not evolved as a field (as for the resistive ballooning model above), the replacement (eФ-3T) $\rightarrow$ eФ applies.

\section{B. The conducting wall model}

For one verification test, name that of the conducting wall mode in the SOL, Eqs. (4) and (5) are replaced by

$$
\begin{gathered}
\frac{\mathrm{c}^{2}}{4 \pi \mathrm{v}_{\mathrm{a}}^{2}}\left(\frac{\partial}{\partial \mathrm{t}}-\mathrm{v}_{\mathrm{E} 0} \cdot \nabla\right) \nabla_{\perp}^{2} \Phi=-\sigma_{\|} \nabla_{\|}^{2} \Phi \\
\left(\frac{\partial}{\partial \mathrm{t}}-\mathrm{v}_{\mathrm{E} 0} \cdot \nabla\right) \mathrm{T}=-\frac{\mathrm{c}}{\mathrm{B}} \mathbf{b} \times \nabla \Phi \cdot \mathbf{e}_{\mathrm{x}} \frac{\partial \mathrm{T}_{0}}{\partial \mathrm{x}}
\end{gathered}
$$

and the full form of the sheath boundary condition, Eq. (6) must be employed. Here 


$$
\mathrm{v}_{\mathrm{E} 0}=\frac{\mathrm{C}}{\mathrm{B}} \mathbf{b} \times \nabla \Phi_{0}
$$

is the equilibrium $\mathrm{E} \times \mathrm{B}$ flow velocity. In this case, for simplicity, the density profile $\mathrm{n}_{0}(\mathrm{x})$ was taken as constant.

\section{Verification tests}

\section{Test 1: Resistive interchange in the edge}

The purpose of this test is to verify the curvature drive term, radial derivative and radial mode structure. For this simple test, a slab geometry is employed, for which the modes obey Eq. (4) with $\sigma_{\|} \nabla_{\|}^{2} \Phi \rightarrow 0$. We chose the dimensionless parameters $\mathrm{R} / \rho_{\mathrm{s}}=$ 1000 , a radial varying density profile $\mathrm{n}_{0}(\mathrm{x})=1-\tanh \left(\mathrm{x} / \mathrm{x}_{\mathrm{W}}\right)+\mathrm{n}_{\mathrm{f}}$ with width $\mathrm{x}_{\mathrm{W}}=10 \rho_{\mathrm{S}}$ on top of a normalized floor density $n_{f}=0.01$. The input wavenumber may be taken as the "binormal” component $\mathrm{k}_{\mathrm{b}}=\mathrm{inB} / \mathrm{RB}_{\theta}$. Results are shown in Fig. 1.
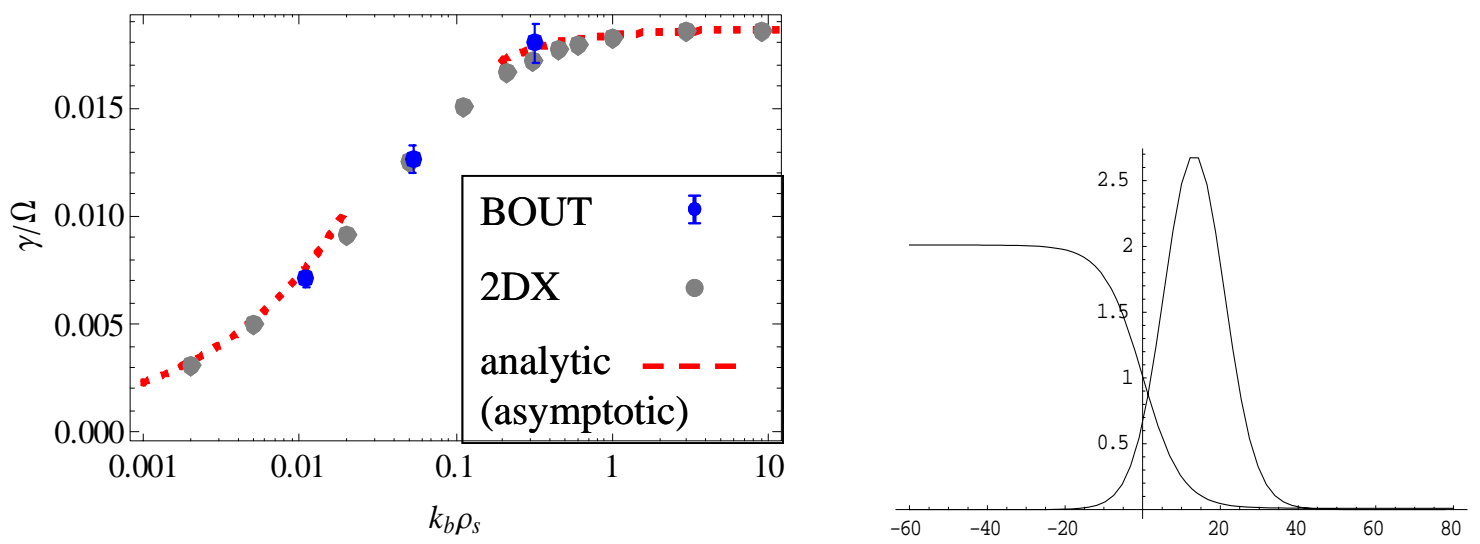

Fig. 1 (left) Dimensionless growth rate $\gamma$ vs. dimensionless $k_{z}$ for the interchange instability with tanh-type density profile. (right) Tanh-type density profile and radial eigenfunction for the case $\mathrm{k}_{\mathrm{b}} \rho_{\mathrm{S}}=0.3(\gamma / \Omega=0.0172)$.

Excellent agreement between the codes and analytical asymptotic results are evident. The analytic curves are obtained by casting the problem into the form

$$
\begin{gathered}
\frac{\mathrm{d}^{2} \Phi}{\mathrm{dx}^{2}}=\left(\mathrm{k}_{\mathrm{z}}^{2}-\frac{\mathrm{d} \alpha}{\mathrm{dx}}\right) \Phi \\
\alpha=\frac{2 \mathrm{k}_{\mathrm{z}}^{2}}{\omega^{2} \mathrm{R}} \ln \mathrm{n}_{0}(\mathrm{x})
\end{gathered}
$$


With $\mathrm{n}_{0}=1-\tanh \left(\mathrm{x} / \mathrm{x}_{\mathrm{w}}\right)+\mathrm{n}_{\mathrm{f}}$, asymptotic solutions for large and small $\mathrm{k}_{\mathrm{z}}$ are obtained as follows:

$$
\gamma^{2}=\left\{\begin{array}{cc}
\frac{\mathrm{k}_{\mathrm{z}}}{\mathrm{R}} \ln \left(\frac{2+\mathrm{n}_{\mathrm{f}}}{\mathrm{n}_{\mathrm{f}}}\right), & \mathrm{k}_{\mathrm{z}} \mathrm{x}_{\mathrm{w}}<1 \\
\frac{2}{\mathrm{RL}_{\mathrm{n}}}, & \mathrm{k}_{\mathrm{z}} \mathrm{x}_{\mathrm{w}}>1
\end{array}\right.
$$

where in Eq. (12) and Fig. 1, $\gamma$ is normalized to the ion cyclotron frequency $\Omega_{\mathrm{i}}$, and $\mathrm{k}_{\mathrm{z}}$ to the Larmor radius (base on the electron temperature) $\rho_{\mathrm{s}}$. It was verified that the

eigenfunctions transition from delta-function-like to tent-function like (i.e. $\mathrm{e}^{ \pm \operatorname{Im} k_{\mathrm{x}} \mathrm{x}}$ ) as $\mathrm{k}_{\mathrm{b}}$ varies from large to small values.

\section{Test 2: Shearless resistive ballooning in the edge}

Next, the resistive ballooning model was solved in an annulus for a circular flux surface geometry in the small inverse aspect ratio limit. The poloidal $(\theta)$ variation of the curvature, $\kappa=-\mathbf{e}_{\mathrm{x}} \cos \theta / \mathrm{R}$, allows testing of the mode structure in $\theta$ and the parallel derivatives in $\sigma_{\|} \nabla_{\|}^{2} \Phi$. It also tests the implementation of phase-shift-periodic boundary conditions for the FLF coordinate system, viz. in order for the physical eigenfunction to be periodic on $\theta=(0,2 \pi)$, the numerical eigenfunction must obey

$$
\Phi(\mathrm{y}=2 \pi)=\Phi(\mathrm{y}=0) \mathrm{e}^{2 \pi \mathrm{in}_{\zeta} \mathrm{q}}
$$

where $\mathrm{q}$ is the safety factor [see Appendix A, Eqs. (A7) and (A12)].

For this test we took $\mathrm{n} \sim \exp \left(-\mathrm{x} / \mathrm{L}_{\mathrm{n}}\right)$ and all equilibrium parameters constant in $\mathrm{x}$ to make the mode structure constant in the radial direction. Parameters were $\mathrm{n}_{\mathrm{e}}=10^{14}$ $\mathrm{cm}^{-3}, \mathrm{~L}_{\mathrm{n}}=9.4 \mathrm{~cm}, \mathrm{~T}_{\mathrm{e}}=100 \mathrm{eV}, \mathrm{R}=202.5 \mathrm{~cm}, \mathrm{~B}=10 \mathrm{kG}, \mathrm{q}=1.5$, Deuterium majority ions, annulus radius $r=0.75 \mathrm{~cm}$, and $Z_{\text {eff }}=32$. The unrealistically large value of $Z_{\text {eff }}$ permits the eigenfunctions to transition between the highly localized limit and the interchange limit. A small $\mathrm{r}$ was chosen to allow accurate comparisons with analytical theory, valid in the limit $\mathrm{r} / \mathrm{R}<<1$. Results are shown in Fig. 2.

The analytic solutions shown in the figure are obtain by first casting the eigenvalue problem for $\gamma$ into the form

$$
\partial_{\mathrm{y}}^{2} \Phi+\frac{\alpha}{\gamma}\left(\gamma_{0}^{2} \cos \mathrm{y}-\gamma^{2}\right) \Phi=0
$$

where

$$
\alpha=\frac{v_{\mathrm{ei}} \mathrm{q}^{2} \mathrm{R}^{2} \mathrm{k}_{\mathrm{z}}^{2}}{2 \Omega_{\mathrm{e}}}
$$




$$
\gamma_{0}^{2}=\frac{2 \mathrm{c}_{\mathrm{s}}^{2}}{\sqrt{\mathrm{RL}_{\mathrm{n}}}}
$$

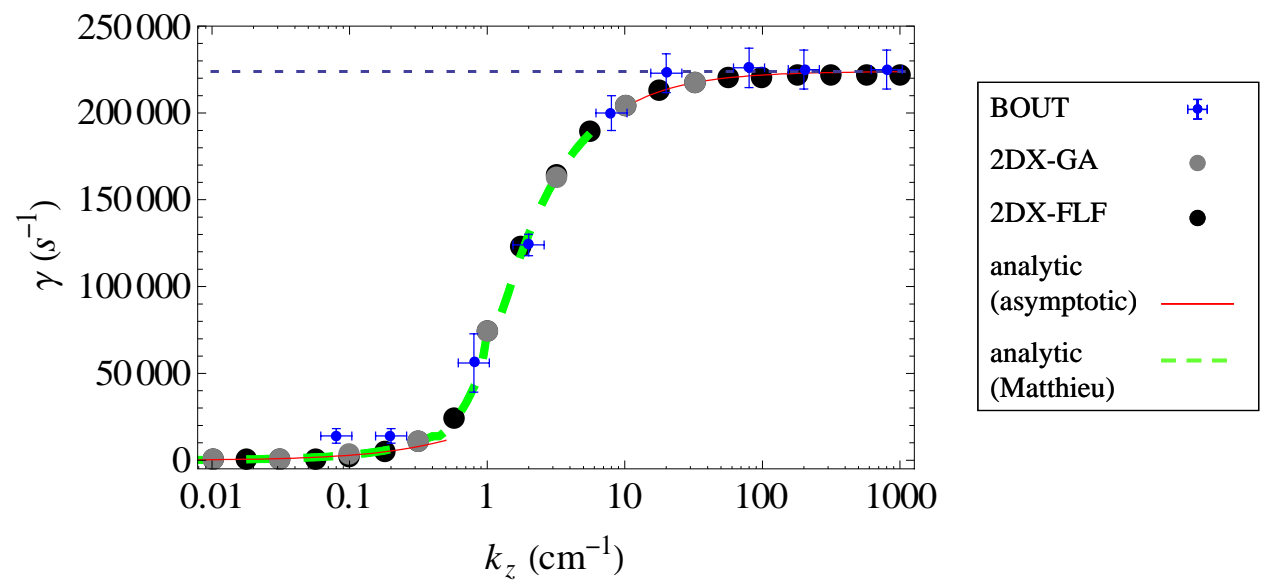

Fig. 2 Growth rate $\gamma$ vs. $k_{Z}$ for the shearless resistive ballooning instability in a shearless annulus. The grey dots are the 2DX code results using GA coordinates and periodic BCs in $\theta$. The black dots are 2DX code results using FLF coordinates and phase-shift-periodic boundary conditions. The crosses are measured growth rates from BOUT with estimated error bars. These numerical results are compared with analytical solutions: the green dashed curve is the numerical solution of the full Mathieu function dispersion relation, the red dashed curves are the small and large $\mathrm{k}_{\mathrm{z}}$ analytical solutions, and, the dotted horizontal line is the local resistive ballooning limit.

Equation (14) may be solved exactly in terms of Mathieu functions; however, not much insight is gained by writing the solution out. In the large $\alpha$ limit, the Mathieu-functions are difficult to calculate, and instead for these cases we employ an analytical closed form solution. To obtain this, we expand $\cos (y)=1-y^{2} / 2$ in Eq. (14) to obtain a Hermite equation. From this it follows that

$$
\gamma=\gamma_{0}-\frac{b \Omega_{i}}{\alpha}
$$

where

$$
\mathrm{b}=\left(\frac{\alpha \gamma_{0}}{8 \Omega_{\mathrm{i}}}\right)^{1 / 2}
$$

In the opposite, small $\alpha$ limit, we transform the unknown from $\Phi$ to $\Psi=\Phi e^{\text {inq } \theta}$ to obtain

$$
\partial_{\theta}^{2} \Phi+2 \operatorname{inq} \partial_{\theta} \Phi-\mathrm{n}^{2} \mathrm{q}^{2} \Phi+\frac{\alpha \gamma_{0}^{2}}{\gamma} \cos \theta \Phi-\alpha \gamma \Phi=0
$$

which is solved for small $\alpha$ by the ansatz $\Phi=1+A \exp (i \theta)$. After some algebra to obtain A, it can be shown that 


$$
\gamma= \begin{cases}\left(\frac{\alpha \gamma_{0}^{4}}{2}\right)^{1 / 3}, & n^{2} q^{2}<<\alpha \gamma \\ \frac{\alpha \gamma_{0}^{2}}{2^{1 / 2} n q} & \alpha \gamma<<n^{2} q^{2}\end{cases}
$$

\section{Test 3: Sheared resistive ballooning in the edge}

To extend the preceding tests, we considered a sheared magnetic field in a plasma slab. This problem couples the radial (x) and parallel (y or $\theta$ ) mode structure. The $q$ profile was taken as $\mathrm{q}=\mathrm{q}_{0}(1+\mathrm{sx} / \mathrm{R})$, and parameters for case \#1 were: $\mathrm{n}_{\mathrm{e}}=10^{14} \mathrm{~cm}^{-3}$, $\mathrm{L}_{\mathrm{n}}=10 \mathrm{~cm}, \mathrm{~T}_{\mathrm{e}}=30 \mathrm{eV}, \mathrm{R}=100 \mathrm{~cm}, \mathrm{~B}=10 \mathrm{kG}, \mathrm{q}_{0}=3, \mathrm{~s}=2.5$, Hydrogen majority ions, $\mathrm{r}=15.92 \mathrm{~cm}, \mathrm{n}_{\zeta}=1$, and $\mathrm{Z}_{\text {eff }}=1$. A second parameter set, case \#2, was also considered with $\mathrm{n}_{\mathrm{e}}=0.5 \times 10^{14} \mathrm{~cm}^{-3}, \mathrm{~L}_{\mathrm{n}}=3.5 \mathrm{~cm}, \mathrm{~s}=2$ and all other parameters the same as for the case \#1. Results are shown in Fig. 3.
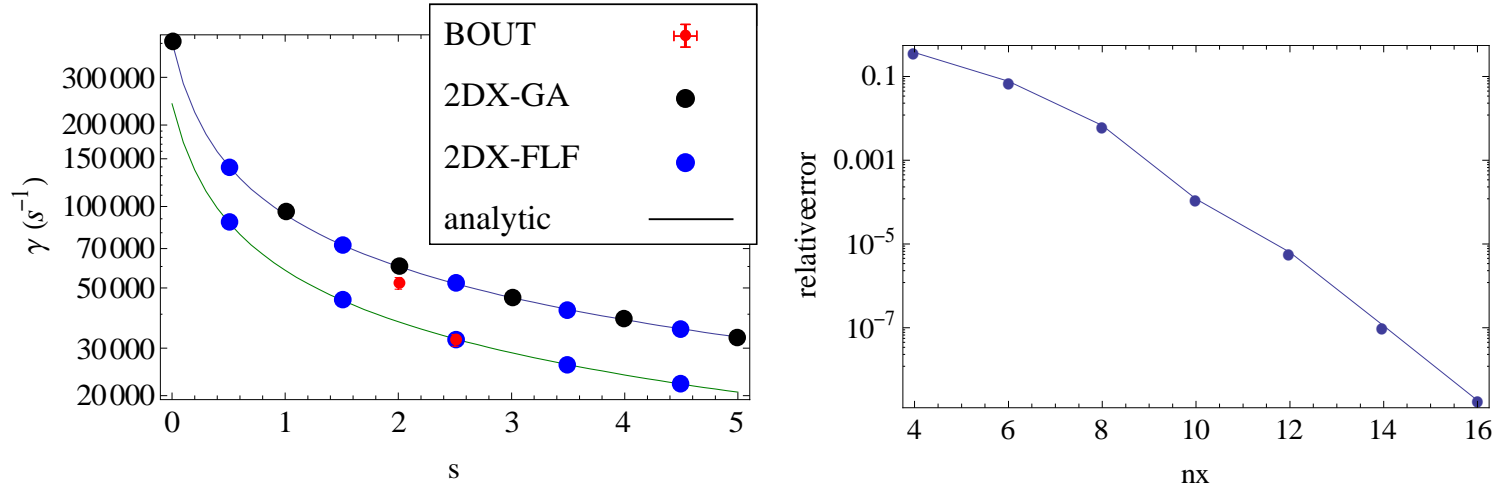

Fig. 3 (left) Growth rate $\gamma$ vs. shear s for the sheared resistive ballooning instability for two test cases. The large dots are 2DX code results, black dots are for GA coordinates, blue dots are for FLF coordinates. The thin lines are the analytical solutions for (green) case \#1 and (blue) case \#2. (right) Convergence study showing relative error in the numerical solution vs. number of radial grid-points.

The analytical solution for this case is obtained following the procedure outlined in Ref. 10. The equivalent eigenvalue problem is cast into the form of a Hermite equation

$$
-\partial_{\mathrm{x}}^{2} \Phi+\mathrm{k}_{\mathrm{b}}^{2} \Phi-\frac{\mathrm{C}_{1}}{\gamma^{2}} \Phi+\frac{\mathrm{C}_{2}}{\gamma} \mathrm{x}^{2} \Phi=0
$$

where here we employ dimensionless Bohm units with $\mathrm{C}_{1}=2 \mathrm{k}_{\mathrm{b}}^{2} / \mathrm{RL}_{\mathrm{n}}$ and $\mathrm{C}_{2}=2 \Omega \mathrm{e}_{\mathrm{b}}^{2} \mathrm{r}^{2} \mathrm{~s}^{2} / \mathrm{v}_{\mathrm{ei}} \mathrm{q}^{2} \mathrm{R}^{4}$. For the parameters of the test cases, it can be shown that the eigenvalues are roots of the cubic

$$
\gamma^{3} C_{2}=\left(C_{1}-\gamma^{2} k_{b}^{2}\right)^{2}
$$


In the limit $\gamma<<\gamma_{0}$, valid for the 2 BOUT test cases, the result is $\gamma=\left(C_{1}^{2} / C_{2}\right)^{1 / 3}$.

Convergence studies were routinely performed on these and other test cases. An example is shown in Fig. 3 (right) which is for the GA coordinates. In this case, the Fourier basis representation was employed. As expected, the convergence is exponentially rapid with increasing mode number.

\section{Test 4: Nedospasov Mode in the SOL}

For the fourth test, we considered a plasma slab in the SOL with sheath boundary conditions. Using the resistive ballooning model, this problem results in the Nedospasov modes, which are basically curvature driven interchange modes made possible by relaxing of line-tying due to the sheaths. ${ }^{12,13}$ The sheath boundary condition in this model is

$$
\nabla_{\|} \Phi= \pm \frac{\mathrm{n}_{0}(\mathrm{x})}{\sigma} \Phi
$$

where the constant parameter $\sigma$ is a normalized Spitzer conductivity. Three test cases were run to allow the boundary condition to transition from a conducting $(\Lambda>>1)$ to an insulating $(\Lambda<<1)$ sheath where $\Lambda=v_{\mathrm{ei}} \mathrm{L}_{\|} / 1.96 \Omega_{\mathrm{e}} \rho_{\mathrm{s}}$. Parameters for case \#1 were: $\mathrm{n}_{\mathrm{e}}=3 \times 10^{13} \mathrm{~cm}^{-3}, \mathrm{~L}_{\mathrm{n}}=4.5 \mathrm{~cm}, \mathrm{~T}_{\mathrm{e}}=5 \mathrm{eV}, \mathrm{R}=202.5 \mathrm{~cm}, \mathrm{~B}=10 \mathrm{kG}, \mathrm{q}=1.5$, Deuterium majority ions, and $Z_{\text {eff }}=1$. Case \#2 and \#3 were the same except for $T_{e}=15$ and $50 \mathrm{eV}$ respectively. Results are shown in Fig. 4. Again good agreement is seen between BOUT, 2DX and the analytical results.

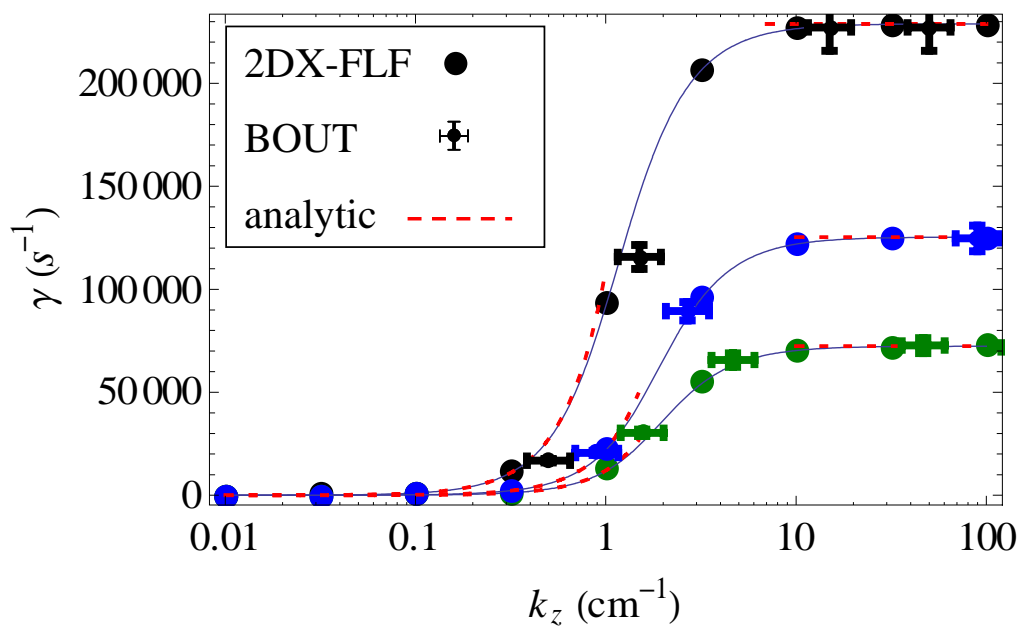

Fig. 4 Test of sheath boundary conditions for Nedospasov-resistive-ballooning problem. Show are growth rates vs. $k_{z}$ for 3 cases, $T_{e}=5,15,50 \mathrm{eV}$ (lower, middle and upper curves). Large dots (respectively green, blue, black) are 2DXev code results. Points with error bars are the BOUT code results. The solid lines are the analytical solution, the dashed red lines are analytic asymptotic limits for small and large $\mathrm{k}_{\mathrm{z}}$. 
The analytical results for this case were obtained by solving the eigenvalue problem

$$
\partial_{\mathrm{y}}^{2} \Phi+\frac{\alpha}{\gamma}\left(\gamma_{0}^{2}-\gamma^{2}\right) \Phi=0
$$

subject to the boundary condition

$$
\partial_{\mathrm{y}} \Phi=\left\{\begin{array}{cc}
\frac{\Lambda}{\mathrm{L}_{\mathrm{y}}} \Phi & \mathrm{y}=\mathrm{y}_{\min }=-\pi \\
-\frac{\Lambda}{\mathrm{L}_{\mathrm{y}}} \Phi & \mathrm{y}=\mathrm{y}_{\max }=\pi
\end{array}\right.
$$

For modes with even parity in $\mathrm{y} \sim \cos \left(\mathrm{k}_{\mathrm{y}} \mathrm{y}\right)$ the solution to this problem is the dispersion relation

$$
\mathrm{k}_{\mathrm{y}}^{2}=\frac{\alpha}{\gamma}\left(\gamma_{0}^{2}-\gamma^{2}\right)
$$

where the $\mathrm{k}_{\mathrm{y}}$ modes are quantize according to $\eta \tan \eta / 2=\Lambda$ with $\eta=\mathrm{k}_{\mathrm{y}} \mathrm{L}_{\mathrm{y}}$ and $\mathrm{L}_{\mathrm{y}}=\mathrm{y}_{\max }$ $-\mathrm{y}_{\min }=2 \pi$. The insulting insulating $(\Lambda<<1)$ and conducting $(\Lambda>>1)$ limits correspond to $\eta=\sqrt{2 \Lambda}$ and $\pi$ respectively.

\section{Test 5: Conducting wall mode in the SOL}

For a final verification test, we considered the "conducting wall" model, sometimes known as the $\nabla \mathrm{T}_{\mathrm{e}}$ sheath instability. ${ }^{14}$ This test requires more complicated boundary condition that couples different fields, $\Phi$ and T, and also yield fully complex eigenvalues. The boundary condition is of the general form

$$
\mathrm{D} \nabla_{\|} \Phi= \pm\left(\Lambda_{1} \Phi-\Lambda_{2} \mathrm{~T}\right)
$$

and for the test cases considered here $\Phi_{0}=\Lambda_{1}=0, \Lambda_{2}=1$ and we used the slab model. Results are shown in Fig. 5. Parameters for case $\# 1$ were $n_{e}=10^{14} \mathrm{~cm}^{-3}, T_{e}=10 \mathrm{eV}, \mathrm{L}_{T}$ $=0.456 \mathrm{~cm}, \mathrm{~B}=10 \mathrm{kG}$, Deuterium majority ions, annulus radius $\mathrm{r}=0.3 \mathrm{~cm}, \ln \Lambda=10$ (Coulomb logarithm) $\mathrm{L}_{\|}=2000 \mathrm{~cm}$, and $\mathrm{Z}_{\text {eff }}=1$. Here, $\mathrm{q}, \mathrm{R}$ and the poloidal domain $\Delta \theta$ only enter through the combination $\mathrm{L}_{\|}=\mathrm{qR} \Delta \theta$ which is the plate-plate distance. Parameters for case \#2 were the same except for $\mathrm{L}_{\mathrm{T}}=500 \mathrm{~cm}$ and a Hydrogen plasma. These two cases cover an extreme range in the temperature gradient scale length $\mathrm{L}_{T}$ which is the controlling parameter for the conducting wall mode. 

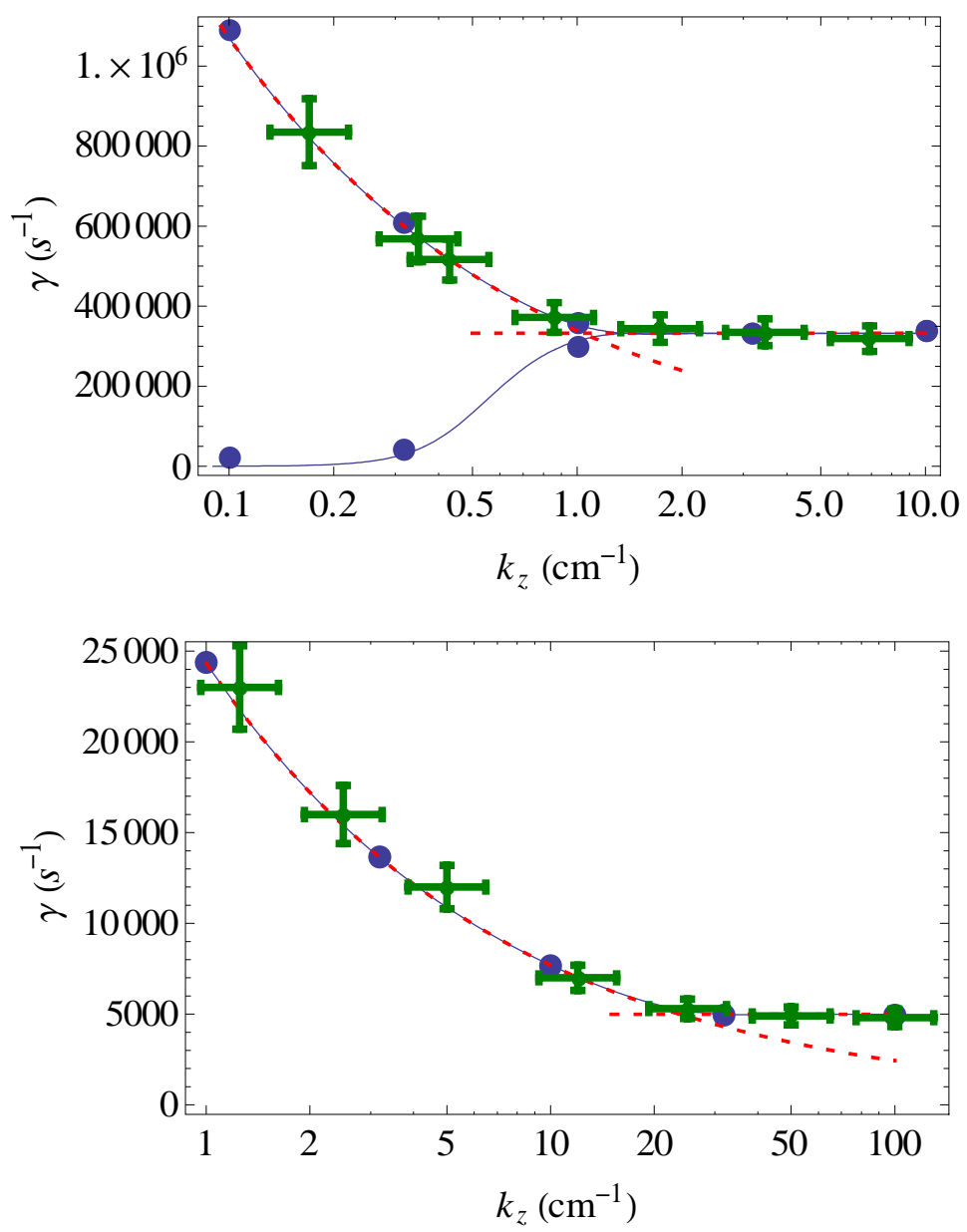

Fig. 5 Test of sheath boundary conditions for the conducting wall mode $\left(\nabla \mathrm{T}_{\mathrm{e}}\right.$ sheath instability $)$ problem. Upper panel: test case \#1, growth rates vs. $\mathrm{k}_{\mathrm{z}}$ for the fastest and 2 nd fastest growing mode. Large dots are 2DX code results. The solid lines are the analytical solution, the dashed red lines are the analytic asymptotic solutions for small and large $\mathrm{k}_{\mathrm{z}}$ for the even mode. Lower panel: same for test case \#2.

The conducting wall mode obeys the following eigenvalue problem

$$
\begin{aligned}
& \left(\partial_{\mathrm{t}}+\mathrm{i} \omega_{\mathrm{E}}\right) \nabla_{\perp}^{2} \Phi=-\mathrm{D} \nabla_{\|}^{2} \Phi \\
& \left(\partial_{\mathrm{t}}+\mathrm{i} \omega_{\mathrm{E}}\right) \mathrm{T}=-\mathrm{i} \mathrm{k}_{\mathrm{b}} \frac{\mathrm{dT}_{0}}{\mathrm{dx}} \Phi
\end{aligned}
$$

where $T$ is the perturbed temperature, the $E \times B$ drift frequency is $\omega_{E}=-k_{b} d \Phi_{0} / d x$, $\mathrm{D}=\sigma \mathrm{T}_{0}^{2} / \mathrm{n}_{0}$. It can be shown that the general dispersion relation is

$$
\tilde{\omega}^{2} \tan \frac{\eta}{2}=-\mathrm{i} \frac{\eta}{2} \omega_{\mathrm{s}}\left(\Lambda_{1} \tilde{\omega}-\Lambda_{2} \omega_{* \mathrm{eT}}\right)
$$


where $\quad \eta=\mathrm{k}_{\|} \mathrm{L}_{\|}, \quad \mathrm{k}_{\|}=(\mathrm{i} \omega / \sigma)^{1 / 2} \mathrm{k}_{\mathrm{b}}, \quad \omega_{\mathrm{s}}=2 / \mathrm{k}_{\mathrm{z}}^{2} \mathrm{~L}_{\|}, \quad \omega_{* \mathrm{eT}}=\mathrm{k}_{\mathrm{z}} \mathrm{dT}_{0} / \mathrm{dx}, \quad$ and $\tilde{\omega}=\omega-\omega_{\mathrm{E}}$. It is straightforward to derive asymptotic forms in the limits of small and large $\mathrm{k}_{\mathrm{z}}$. For small $\mathrm{k}_{\mathrm{z}}$ we let $\tan (\eta / 2) \approx \eta / 2$ and immediately obtain from Eq. (30) a quadratic dispersion relation in $\omega$. For large $\mathrm{k}_{\mathrm{z}}$, anticipating $\operatorname{large} \operatorname{Im}(\eta)$ we let $\tan (\eta / 2) \approx$ i to obtain $\gamma=-\mathrm{i} \omega=\exp (\mathrm{i} \pi / 3) /\left(\sigma^{1 / 3} \mathrm{~L}_{\mathrm{T}}^{2 / 3}\right)$ where $1 / \mathrm{L}_{\mathrm{T}}=-\mathrm{d} \ln \mathrm{T}_{0} / \mathrm{dx}$ where for simplicity we consider the test case with $\Phi_{0}=\Lambda_{1}=0, \Lambda_{2}=1$.

Finally, we note that the radial mode structure for the conducting wall mode has also been studied in Refs. 15 and 16 where additional analytical limits and tests are available.

\section{Extensions of the 2DX code}

\section{A. Mixed magnetic topology}

The preceding section has demonstrated a number of verification tests of the BOUT and 2DX codes. These have been in geometries where the magnetic field has a simple topology. BOUT has the capability of describing full X-point/divertor geometry, and this capability has recently been implemented in 2DX, in a phased development.

As a first step, in the circular flux surface model, we considered a domain which includes both closed and open field line regions, and successfully implemented the correct parallel boundary conditions in each region (phase-shift-periodic and sheath respectively), and matched the solutions radially across the last closed surface (LCS). This version of the code reproduces all previous test results in appropriate limits. It also enables the description of other interesting cases such as LCS-spanning modes. Two examples are shown in Fig. 6, both employing the resistive ballooning model. One case illustrates a mode localized near the LCS but primarily in the SOL because of the insulting sheath boundary conditions which are inherently more unstable. A second example shows a mode that is line-tied in the SOL (conducting limit of the sheath boundary condition) but interchange-like in the edge. Since the conducting boundary condition is stabilizing, the mode favors the edge over the SOL. These cases demonstrate handling of mixed magnetic topologies. Full X-point/divertor geometry is considered in Sec. V-C. 

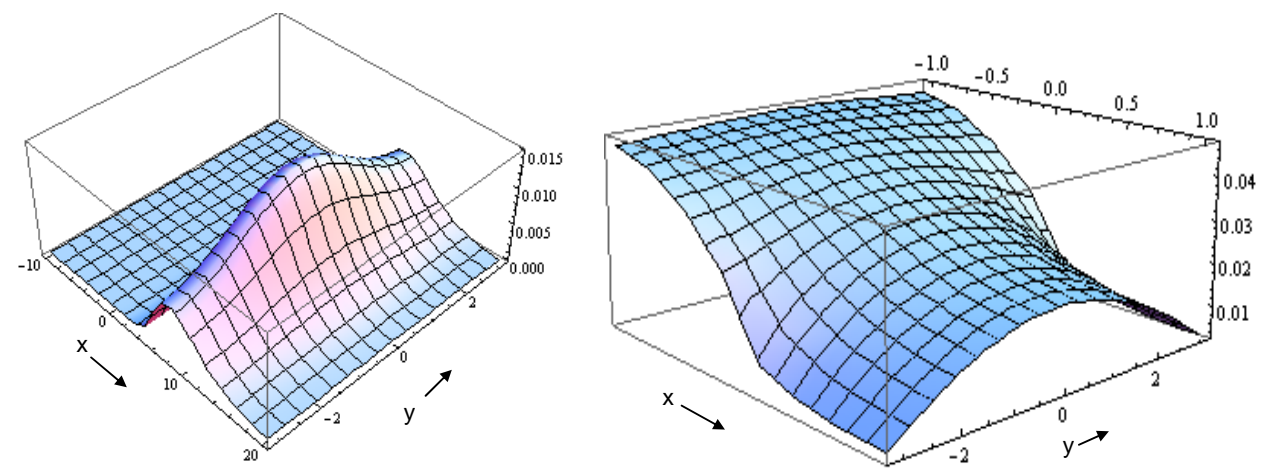

Fig. 6 (left) Eigenfunction $\Phi$ for a mode that favors the SOL (insulating sheath boundary condition limit $\nabla_{\|} \Phi=0$ ) and (right) one that favors the edge (conducting sheath boundary condition limit $\Phi=0)$. These cases demonstrate handling of mixed magnetic topologies. Here the last closed surface is at $x=0$, the SOL is $x>0$, and $\theta$ runs from $-\pi$ to $\pi$ where $\theta=0$ is the outboard midplane where the curvature drive is strongest.

\section{B. Kinetic physics}

The eigenvalue method employed by the 2DX code is amenable to extensions that can allow modeling of kinetic effects on boundary plasma modes. We present here a proof-of-principle demonstration. For the physics model, we replace the Spitzer conductivity $\sigma_{\|}$in the resistive ballooning equations of Eq. (4) by the electron kinetic conductivity

$$
\sigma_{\|}=\frac{\omega}{4 \pi \mathrm{i}} \frac{1}{\mathrm{k}_{\|}^{2} \lambda_{\mathrm{de}}^{2}}(1+\zeta \mathrm{Z}(\zeta))
$$

where $\mathrm{Z}(\zeta)$ is the usual plasma dispersion function ${ }^{17}$ and here we take

$$
\zeta=\frac{\omega+\mathrm{i} v_{\mathrm{ei}} / 1.96}{\left|\mathrm{k}_{\|}\right| \sqrt{2} \mathrm{v}_{\mathrm{e}}}
$$

This model has the property that it recovers the Spitzer conductivity in the collisional limit $v_{\mathrm{ei}}>\omega$, and reduces to the usual collisionless result in the opposite limit. Furthermore, by retaining $v_{\mathrm{ei}} \sim \omega$ but expanding the Z-function for $\zeta>>1$, the model yields the Braginskii result with electron inertia (from the $\omega$ ) in addition to Spitzer conductivity (from the $v_{\mathrm{ei}}$ ). ${ }^{18}$ The point here is not the validity of the model, which can be questioned, but rather the demonstration of a numerical technique for treating kinetic effects which introduce matrix elements that (i) depend nonlinearly on the eigenvalue $\omega$, and (ii) depend on transcendental functions of spatial derivate operators like $\mathrm{k}_{\|}$(giving, in effect, integral equations in configuration space). 
Our numerical method is to obtain the matrix $\mathrm{M}(\gamma)$ and iterate $\mathrm{M}(\gamma) \xi=\gamma \xi$ to convergence. For this, we use eigenvalue perturbation theory ${ }^{19}$ together with a Newtontype iteration. The algorithm for obtaining the next refinement of $\gamma$, viz. $\gamma_{1}$ given a guess $\gamma_{0}$ is

$$
\gamma_{1}=\gamma_{0}-\frac{F\left[M\left(\gamma_{0}\right)\right]-\gamma_{0}}{\left\langle\mathrm{x}_{0} \mid \frac{\partial \mathrm{M}}{\partial \gamma} \mathrm{x}_{0}\right\rangle-1}
$$

where $\mathrm{F}[\mathrm{M}]$ is the largest eigenvalue (fastest mode) of the matrix $\mathrm{M}$, and the $<\ldots \mid \ldots>$ notation denotes an inner product.

For this test we employ GA coordinates on the closed surfaces, with the Fourier basis set. This allows the Z-function matrix elements involving $\mathrm{k}_{\|}$to be formed in Fourier space. The matrix operator representation in Fourier space is then transformed back to configuration space.

Figure 7 illustrates results for the following parameter set (realistic, but somewhat optimized to illustrate the role of kinetic effects): $\mathrm{n}_{\mathrm{e}}=10^{13} \mathrm{~cm}^{-3}, \mathrm{~L}_{\mathrm{n}}=4 \mathrm{~cm}, \mathrm{R}=202.5$ $\mathrm{cm}, \mathrm{B}=3.10^{4} \mathrm{G}, \mathrm{q}=3.3$, Deuterium ions, $\mathrm{Z}_{\mathrm{eff}}=1$ and $\mathrm{n}_{\zeta}=5$.

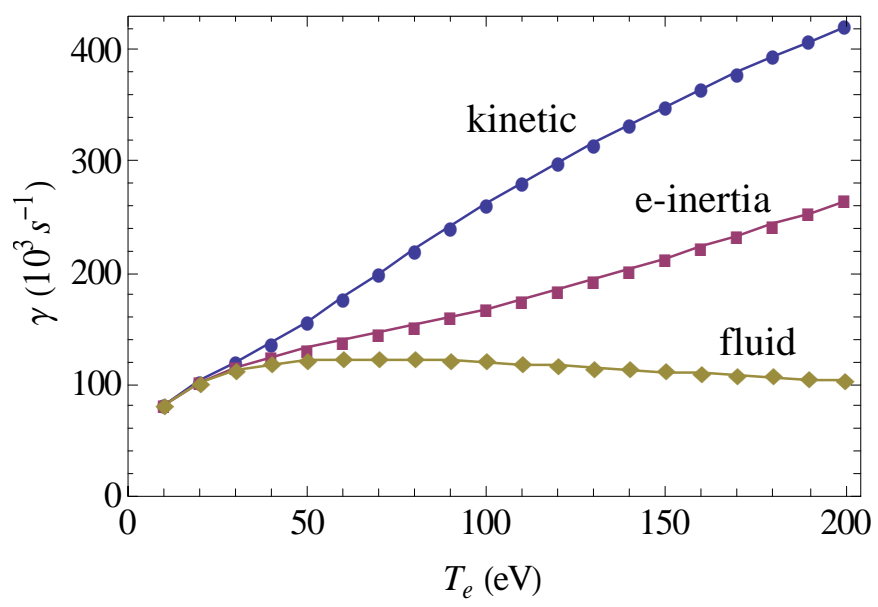

Fig. 7 Growth rate $\gamma\left(10^{3} / \mathrm{s}\right)$ vs. $\mathrm{T}_{\mathrm{e}}(\mathrm{eV})$ for the three cases: kinetic (top blue curve), fluid with electron inertia (middle plum curve) and collisional fluid (lower olive curve). Note that for small $\mathrm{T}_{\mathrm{e}}$ all the models are equivalent, but at large $\mathrm{T}_{\mathrm{e}}$ kinetic physics adds additional instability drive.

\section{File sharing and full divertor geometry}

In the initial development of the 2DX code, magnetic geometry information, such as $B$, the safety factor $\mathrm{q}$, and shear $\mathrm{s}$ where input as analytical functions. This has now been extended to enable complex magnetic geometry to be read from an HDF file. Typically the flux surface geometry is obtained from the UEDGE code. Routines have 
been written to translate the equilibrium PDB files produced by UEDGE into HDF format. The datasets used by 2DX specify $\mathrm{R}(\psi, \theta), \mathrm{Z}(\psi, \theta), \mathrm{B}_{\mathrm{t}}(\psi, \theta)$ on a $\psi, \theta$ grid from which all other magnetic geometry quantities can be calculated. BOUT performs similar preprocessing on the UEDGE code 20 output PDB files. Equilibrium plasma profiles are all in the PDB and HDF files. Thus 2DX and BOUT get the same input. Independent processing of subsidiary magnetic geometry quantities (e.g. the local safety factor, or the components of the curvature vector) by 2DX and BOUT provides a verification check on these preprocessor calculations. File sharing of complex magnetic geometries such as divertor X-point configurations, gives 2DX the same geometry capability as BOUT.

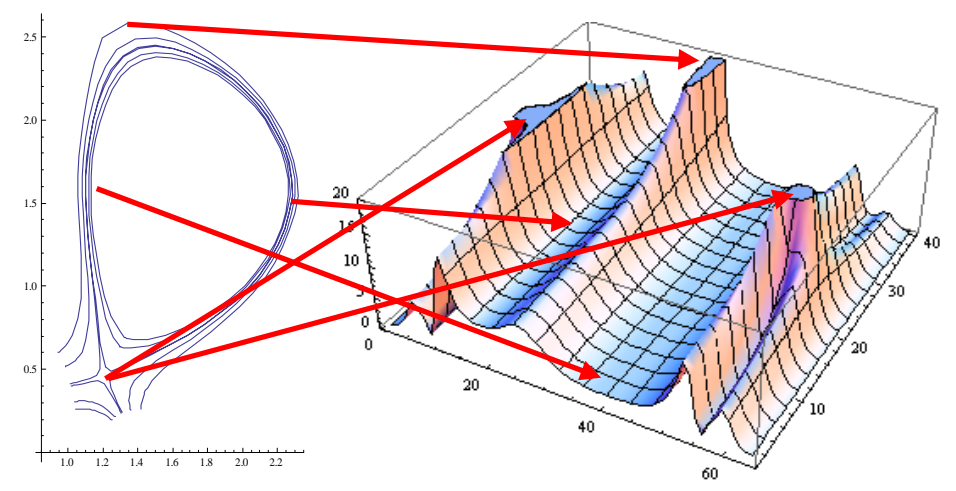

Fig. 8 Sample edge magnetic geometry showing the flux surface shape and the radial and poloidal variation of the local safety factor $v$ for a DIII-D equilibrium.
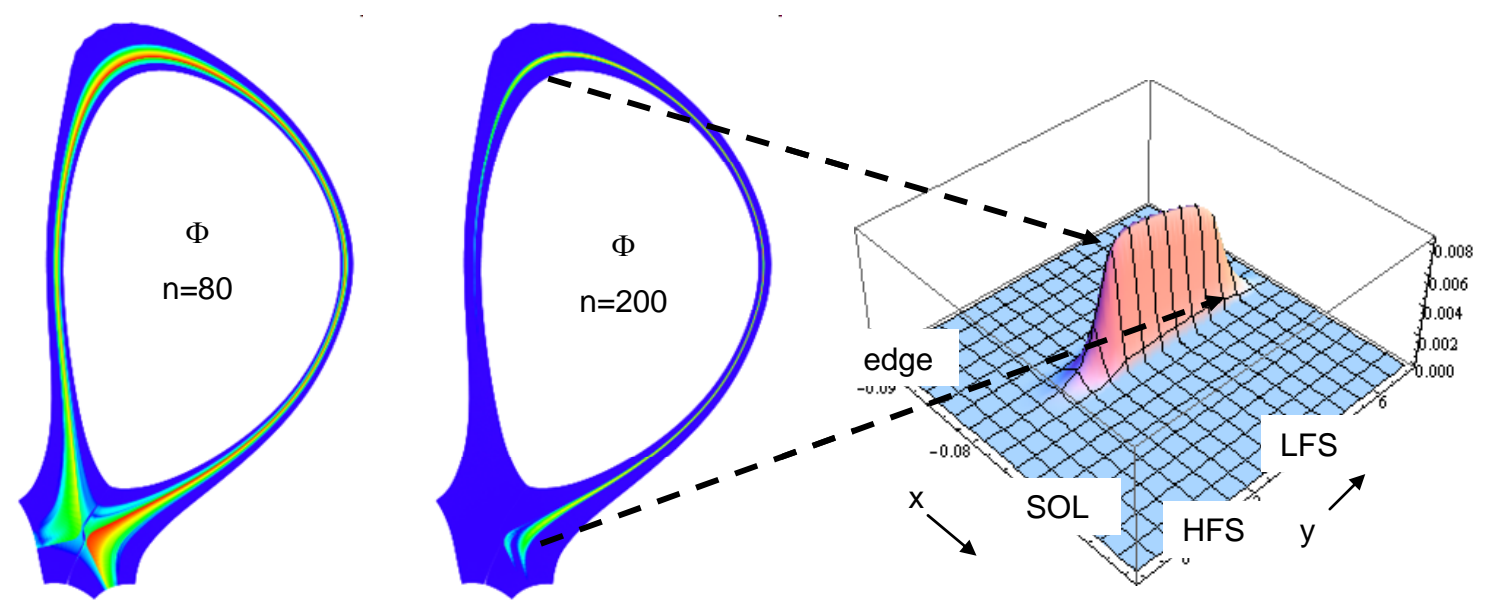

Fig. 9 Demonstration of realistic divertor geometry in 2DX. Shown is a DIII-D lower-single-null case. Left to right, the panels are: $n=80$ resistive ballooning eigenmode $\Phi$ (logarithmic color palette in the $R, Z$ plane), $n=200$ eigenmode $\Phi$, and the same $n=200$ eigenmode on the computational grid. 
For the DIII-D lower-single-null case of Fig. 8, 2DX solutions of the resistive ballooning model are illustrated in Fig. 9. Several physics points emerge from these solutions. In particular, note that the $n=200$ mode is confined poloidally between the lower X-point and a virtual X-point (in the vacuum outside the computational domain), while the $n=80$ mode can tunnel through the X-points and actually reaches the lowfield-side divertor plate. As expected, the $n=200$ mode is also radially narrower.

\section{Summary}

A series of successful benchmark tests has been carried out between BOUT and the new edge eigenvalue code 2DX. The results shown here extend earlier verification studies for BOUT, ${ }^{21}$ provide verification of $2 \mathrm{DX}$, and are a step towards the creation of accepted verification standards for edge turbulence codes by considering growth rates of "global” edge-SOL modes in tokamak divertor geometry.

Concerning 2DX, there are several noteworthy features regarding its development. It appears both feasible and useful to implement both geometric angle and field-linefollowing coordinates. This feature may provide further verification checks, important for complex magnetic topologies, and would allow for increased computational efficiency by enabling the end user to make an optimal choice for the problem at hand. 2DX has been developed using good coding practices. The 2D eigenvalue approach permits rather simple, modular coding, and considerable flexibility in numerical schemes for differential (and integral) operators. Finally, proof-of-principle kinetic extensions to the model two-fluid have been demonstrated in 2DX.

Future plans for this work include development of a more automated suite of benchmarked cases for BOUT and 2DX with full documentation, so that these cases can serve as standards for other edge codes. 2DX requires a number of additional development steps to reach its full potential. These include implementation of double Xpoint magnetic topology, and more comprehensive physics models with evolution equations for $\mathrm{A}_{\|}, \mathrm{u}_{\|}, \mathrm{T}_{\mathrm{e}}, \mathrm{T}_{\mathrm{i}}$, and $\mathrm{J}_{\|}$. Ultimately electron kinetic physics for these moments is desirable as well as kinetic ion FLR orbits. Some of the combinations of options discussed in this report have not yet been fully implemented, such as choices for the differential operator representations, coordinate systems, boundary conditions, and topologies.

The present proof-of-principle version of the 2DX code employs a standard fullmatrix package for the eigen-solutions. While this permits the use of basis sets with noncompact support such as Fourier modes, and gives high order accuracy, it also places large demands on the system memory for the computation. When memory requirements limit the desired resolution, sparse vs. full matrix storage and the use of finite difference representations becomes attractive. Sparse matrix algorithms are then required which can 
extract the eigenvalue corresponding to the fastest growing mode, i.e. $\operatorname{Max}(\operatorname{Re}(\gamma))$, or if necessary the complete eigenvalue spectrum. Several methods including the Arnoldi method are possible. Initial tests of an iterative algorithm that is essentially an implicit, centered time-stepping scheme, appear to work quite well in extracting the fastest growing mode. Some further development along these lines is required. Finally, translation of the present code, written in Mathematica, to a public domain language (probably Fortran9x) is planned.

\section{Acknowledgments}

This work was supported by the U.S. Department of Energy under DOE/SBIR grant DE-FG02-07ER84718, and (at LLNL) was performed under the auspices of the U.S. Department of Energy by Lawrence Livermore National Laboratory under Contract DE-AC52-07NA27344. This support does not constitute an endorsement by the DOE of the views expressed herein.

\section{Appendix A: Coordinate systems and differential operators}

Two coordinate systems are proto-typed in the 2DX code, geometrical-angle (GA) coordinates, and field-line-following (FLF) coordinates. The simplest case is GA for which the coordinates are

$$
\begin{aligned}
& \mathrm{x}=\psi-\psi_{0} \\
& \mathrm{y}=\theta \\
& \mathrm{z}=\zeta
\end{aligned}
$$

Exploiting toroidal symmetry by performing a toroidal mode expansion, the mode in these coordinates is represented as

$$
\Phi=\Phi_{\mathrm{GA}}(\mathrm{x}, \mathrm{y}) \exp (\mathrm{in} \zeta)
$$

where in this appendix, $\mathrm{n}$ is the toroidal mode number, and $\Phi_{\mathrm{GA}}(\mathrm{x}, \mathrm{y})$ is the function that is being solved for numerically. The physics usually dictates that $\mathrm{k}_{\|}$is small compared with $\mathrm{k}_{\perp}$, where

$$
\mathrm{k}_{\|} \sim \frac{\mathrm{nq}-\mathrm{m}}{\mathrm{qR}}
$$

and $\mathrm{m}$ represents a typical mode number in the poloidal direction, i.e. $\mathrm{k}_{\perp} \sim \mathrm{m} / \mathrm{r}$. Thus GA coordinates have the disadvantage that for large $n, \Phi_{\mathrm{GA}}(\mathrm{x}, \mathrm{y})$ can be rapidly varying in $\mathrm{y}$, and this structure must be resolved numerically. The principle advantage of GA coordinates is their conceptual simplicity and a relatively straightforward (though perhaps messy, because of metric tensor elements) implementation of operators and 
boundary conditions. In GA coordinates, taking as an example the case where $\nabla \theta$ and $\nabla \psi$ are orthogonal, and $\nabla_{\|}<<\nabla_{\perp}$ we have

$$
\begin{gathered}
\nabla_{\|}=\frac{\operatorname{inB}_{\zeta}}{\mathrm{BR}}+\frac{1}{v \mathrm{R}} \frac{\partial}{\partial \theta} \\
\nabla_{\perp}^{2} \approx \nabla^{2}=\frac{1}{\mathrm{~J}} \frac{\partial}{\partial \psi} \mathrm{J}|\nabla \psi|^{2} \frac{\partial}{\partial \psi}+\frac{1}{\mathrm{~J}} \frac{\partial}{\partial \theta} \mathrm{J}|\nabla \theta|^{2} \frac{\partial}{\partial \theta}-\frac{\mathrm{n}^{2}}{\mathrm{R}^{2}}
\end{gathered}
$$

with $v(\mathrm{x}, \mathrm{y})$ the local safety factor and $\mathrm{J}^{-1}=\nabla \psi \times \nabla \theta \cdot \nabla \zeta$. Generalization to the nonorthogonal case is straightforward. Obviously, with the representation of Eq. (A2), $\Phi$ is periodic in $\theta$ when $\Phi_{\mathrm{GA}}$ is periodic in $\mathrm{y}$.

The FLF choice for coordinates is slightly more complicated. In this case, the coordinates are defined by

$$
\begin{aligned}
& x=\psi-\psi_{0} \\
& y=\theta \\
& z=\zeta-\int_{\theta_{0}}^{\theta} d \theta v
\end{aligned}
$$

where $\theta_{0}$ is an arbitrary constant. Again invoking the toroidal mode expansion, it can be shown that using FLF coordinates is equivalent to assuming a mode representation of the form

$$
\Phi=\Phi_{\mathrm{FLF}}(\mathrm{x}, \mathrm{y}) \exp \left(\operatorname{in} \zeta-\operatorname{in} \int_{\theta_{0}}^{\theta} \mathrm{d} \theta \nu\right)
$$

where $\Phi_{\mathrm{FLF}}(\mathrm{x}, \mathrm{y})$ is the function that is being solved for numerically. When $\mathrm{k}_{\|}<<\mathrm{k}_{\perp}$, FLF coordinates insure that $\Phi_{\mathrm{FLF}}(\mathrm{x}, \mathrm{y})$ is slowly varying even for large $\mathrm{n}$. Basically, the $\mathrm{y}$ dependence corresponds to $\mathrm{k}_{\|}$and the fast $\theta$ dependence has been extracted into the phase factor in Eq. (A7). In FLF coordinates, the representation for the differential operators is

$$
\begin{gathered}
\nabla_{\|}=\frac{1}{\nu \mathrm{R}} \frac{\partial}{\partial \mathrm{y}} \\
\nabla_{\perp}^{2}=-\frac{1}{\mathrm{~J}}\left(\mathrm{k}_{\psi}-\mathrm{i} \partial_{\mathrm{x}}^{\mathrm{a}}\right) \mathrm{J}\left(\mathrm{k}_{\psi}-\mathrm{i} \partial_{\mathrm{x}}^{\mathrm{b}}\right)-\mathrm{k}_{\mathrm{b}}^{2}
\end{gathered}
$$

where

$$
\begin{gathered}
\mathrm{k}_{\mathrm{b}}=-\mathrm{nB} / \mathrm{RB}_{\theta} \\
\mathrm{k}_{\psi}=-\mathrm{n}|\nabla \psi|\left(\frac{v \nabla \theta \cdot \nabla \psi}{|\nabla \psi|^{2}}+\int_{\theta_{0}}^{\theta} \mathrm{d} \theta \frac{\partial v}{\partial \psi}\right)
\end{gathered}
$$

and for any quantity Q 


$$
\begin{aligned}
& \partial_{\mathrm{x}}^{\mathrm{a}} \mathrm{Q}=\frac{\partial}{\partial \psi}|\nabla \psi| \mathrm{Q} \\
& \partial_{\mathrm{x}}^{\mathrm{b}} \mathrm{Q}=|\nabla \psi| \frac{\partial}{\partial \psi} \mathrm{Q}
\end{aligned}
$$

It is important to note the integrals in Eqs. (A6), (A7) and (A11). These field line integrals can become discontinuous across a separatrix, because they depend on integrands which may be very different in topologically separate regions. An example is the separatrix delimiting the main SOL from the private SOL in divertor geometry.

Finally, note that with the FLF representation of Eq. (A7), $\Phi$ is periodic in $\theta$ when $\Phi_{\mathrm{FLF}}$ obeys the phase-shift-periodic boundary condition

$$
\Phi_{\mathrm{FLF}}(\mathrm{y}=0)=\Phi_{\mathrm{FLF}}(\mathrm{y}=2 \pi) \mathrm{e}^{-2 \pi \mathrm{inq}}
$$

where

$$
q=\frac{1}{2 \pi} \int_{0}^{2 \pi} d \theta v
$$

\section{Appendix B: 2DX code architecture and implementation}

The 2DX code invokes the following modular steps:

(1) Set up coordinate grids in $\mathrm{x}$ and $\mathrm{y}$.

(2) Define elementary $1 \mathrm{D}$ operators, e.g. $\partial_{\mathrm{X}}$ as $\mathrm{n}_{\mathrm{X}} \times \mathrm{n}_{\mathrm{X}}$ sub-matrices. This is done for each projection method (Fourier or spatial discretization).

(3) Construct operators in the 2D space using a flattened-outer-product, e.g. $\partial_{\mathrm{x}}^{2 \mathrm{D}}=\partial_{\mathrm{x}}^{1 \mathrm{D}} \bar{\otimes} \mathrm{I}_{\mathrm{y}}$ where Iy is the identity matrix on the y-grid.

(4) From the elementary operators, such as $\partial_{x}$ and $\partial_{y}$, build up operators required for the physics model, such as $\nabla_{\perp}, \nabla_{\|}$. This is done for each coordinate system, GA and FLF.

(5) Build each sub-matrix $M_{i j}$, where $i$, j runs over all the fields, e.g. $\Phi, n$ as illustrated in Eq. (3).

(6) Build the total matrix $M$ from the sub-matrices.

(7) Invoke the eigenvalue solver

(8) If desired, reconstruct the eigenfunctions for the field(s) on the 2D grid

In step (3), the flattened-outer-product $\bar{\otimes}$ is an operation which creates 2D operators from 1D subspaces. Defining this operation allows almost index-free coding. 
Rather than given a formal definition, consider the following simple example. Given 1D operators $\mathrm{X}$ and $\mathrm{Y}$

$$
X=\left(\begin{array}{ll}
X_{11} & X_{12} \\
X_{21} & X_{22}
\end{array}\right), \quad Y=\left(\begin{array}{cc}
Y_{11} & Y_{12} \\
Y_{21} & Y_{22}
\end{array}\right)
$$

the outer product is given by

$$
X \bar{\otimes} Y \equiv\left(\begin{array}{llll}
X_{11} Y_{11} & X_{11} Y_{12} & X_{12} Y_{11} & X_{12} Y_{12} \\
X_{11} Y_{21} & X_{11} Y_{22} & X_{12} Y_{21} & X_{12} Y_{22} \\
X_{21} Y_{11} & X_{21} Y_{12} & X_{22} Y_{11} & X_{22} Y_{12} \\
X_{21} Y_{21} & X_{21} Y_{22} & X_{22} Y_{21} & X_{22} Y_{22}
\end{array}\right)
$$

Use of the flattened outer produce facilitates intermixing of various options. For example finite difference and Fourier representations of the $1 \mathrm{D}$ second-derivative operation can be defined respectively as

$$
\partial_{\mathrm{x}}^{2} \equiv\left(\begin{array}{cccc}
-2 / \Delta^{2} & 1 / \Delta^{2} & 0 & 0 \\
1 / \Delta^{2} & -2 / \Delta^{2} & 1 / \Delta^{2} & 0 \\
0 & 1 / \Delta^{2} & -2 / \Delta^{2} & 1 / \Delta^{2} \\
0 & 0 & 1 / \Delta^{2} & -2 / \Delta^{2}
\end{array}\right)
$$

and

$$
\partial_{\mathrm{X}}^{2} \equiv-\mathrm{Q}^{-1} \mathrm{k}_{\mathrm{X}}^{2} \mathrm{Q}
$$

where $\mathrm{Q}_{\mathrm{ij}}=\exp \left[-\mathrm{ik}_{\mathrm{i}}\left(\mathrm{x}_{\mathrm{j}}-\mathrm{x}_{\text {mid }}\right)\right]$. Multiple representations for operation in the $1 \mathrm{D} \mathrm{y}$ subspace are similarly define. Any $2 \mathrm{D}$ combination of these is then readily constructing using the $\bar{\otimes}$ operator.

\section{References}

1 X.Q. Xu and R.H. Cohen, Contrib. Plasma Phys. 36, 158 (1998).

2 M.V. Umansky et al., Contrib. Plasma Phys. 44, 182 (2004).

3 S. J. Zweben, J. A. Boedo, O. Grulke, C. Hidalgo, B. LaBombard, R. J. Maqueda, P. Scarin and J. L. Terry, Plasma Phys. Control. Fusion 49, S1 (2007).

4 J.R. Myra and D. A. D’Ippolito, Phys. Plasmas 12, 092511 (2005).

5 S. I. Krasheninnikov, D. A. D’Ippolito and J. R. Myra, "Review article: Recent Theoretical Progress in Understanding Coherent Structures in Edge and SOL Turbulence”, J. Plasma Phys (2008), to be published.

6 P.W. Terry , M. Greenwald , J.-N. Leboeuf, G.R. McKee, D.R. Mikkelsen , W.M. Nevins, D.E. Newman, and D.P. Stotler, "Validation in Fusion Research: Towards Guidelines and Best Practices,” eprint arXiv/0801.2787. 
7 J. R. Myra, J. Boedo, B. Coppi, D. A. D’Ippolito, S. I. Krasheninnikov, et al., in Plasma Physics and Controlled Nuclear Fusion Research 2006 (IAEA, Vienna, 2007), paper IAEACN-149-TH/P6-21.

8 P. B. Snyder, H. R. Wilson, J. R. Ferron, L. L. Lao, et al., Phys. Plasmas 9, 2037 (2002); and refs. therein.

9 J. R. Myra, Phys. Plasmas 14, 102314 (2007); and refs. therein.

10 B. A. Carreras, L. Garcia and P. H. Diamond, Phys. Fluids 30, 1388 (1987).

11 J.W. Connor, R.J. Hastie, and J.B. Taylor, Phys. Rev. Lett. 40, 396 (1978).

12 A. V. Nedospasov, Fiz. Plazmy 15, 1139 (1989) [Sov. J. Plasma Phys. 15, 659 (1989)].

13 X. Garbet, L. Laureent, J.-P. Roubin, and A. Samain, Nucl. Fusion 31, 967 (1991).

14 H. L. Berk, R. H. Cohen, D. D. Ryutov, Yu. A. Tsidulko, and X. Q. Xu, Nucl. Fusion 33, 263 (1993).

15 K. Lotov, D. Ryutov and J. Weiland. Phys. Scripta 50, 153 (1994).

16 D.R. McCarthy, P.J. Catto, S.I. Krasheninnikov. Phys. Plasmas 8, 750 (2001).

17 B.D. Fried and S.D. Conte, The Plasma Dispersion Function, (Academic Press, New York, 1961).

18 S. I. Braginskii, in Reviews of Plasma Physics, edited by M. A. Leontovich (Consultants Bureau, New York, 1965), Vol. 1, p. 205.

19 P. Stehle, Quantum Mechanics (Holden-Day, San Francisco, 1966), pp. 123 - 126; or see any standard textbook on quantum mechanics.

20 T. D. Rognlien, J. L. Milovich, M. E. Rensick, and G. D. Porter, J. Nucl.Mater. 196, 347 (1992).

21 M. V. Umansky et al., "Suite of Verification Test Problems for Edge Turbulence Simulations” to be published in Contrib. Plasma Phys. 